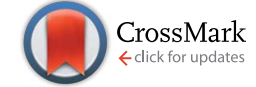

Cite this: RSC Adv., 2017, 7, 2315

Received 21st October 2016 Accepted 14th December 2016

DOI: $10.1039 / c 6 r a 25627 a$

www.rsc.org/advances

\section{A novel ionic liquid functionalized graphene oxide supported gold nanoparticle composite film for sensitive electrochemical detection of dopamine}

\author{
Jianbo Li, Yanhui Wang, Yuanling Sun, Chaofan Ding, Yanna Lin, Weiyan Sun \\ and Chuannan Luo*
}

A simple and sensitive electrochemical sensor for detection of dopamine has been developed based on ionic liquid functionalized graphene oxide supported gold nanoparticles (GO-IL-AuNPS) coated onto a glassy carbon electrode. The prepared graphene oxide, ionic liquid functionalized graphene oxide and GO-IL-AuNPs were characterized in detail by scanning electron microscopy, X-ray diffraction and Fourier transform infrared spectroscopy. The electrochemical properties of the hybrid nanomaterials were evaluated through cyclic voltammetry and electrochemical impedance spectroscopy. Meanwhile, the activities of hybrid nanomaterials toward the oxidation of dopamine were analyzed via cyclic voltammetry and differential pulse voltammetry. The resulting sensor showed excellent electrocatalytic activity towards dopamine. Under the optimum conditions, differential pulse voltammetry was employed to detect ultra-trace amounts of dopamine, for which a wide linear range of $7 \mathrm{nM}$ to $5 \mu \mathrm{M}$ and a limit of detection $2.3 \mathrm{nM}(\mathrm{S} / \mathrm{N}=3)$ were obtained. The proposed sensor exhibited excellent accuracy and precision and the relative standard deviation (RSD) was less than $5 \%$. The highly sensitive sensor was successfully used for accurate determination of the content of dopamine in practical samples.

\section{Introduction}

Dopamine (DA) is a catecholamine neurotransmitter secreted by the brain that plays a critical role in maintaining the functional activities of many organs, such as the heart, brain and kidney. ${ }^{1-3}$ Dopamine can directly affect human emotions, learning and memory ability. Meanwhile, as a very important neurotransmitter, it mainly exists in the body fluid and brain cells. Research indicates that abnormal levels of dopamine are linked to some ailments, such as Alzheimer's disease, depression, Parkinson's disease, hyperactivity disorder and schizophrenia. ${ }^{4-7}$ Hence, the rapid and accurate measurement of the content of DA is a significant research area in clinical practice. So far, many analytical approaches have been developed for detection of DA, including high performance liquid chromatography, ${ }^{8}$ fluorescence, ${ }^{9}$ chemiluminescence, ${ }^{\mathbf{1 0}}$ mass-spectrometry, ${ }^{11}$ capillary-electrophoresis, ${ }^{12,13}$ and surface plasmon resonance. ${ }^{\mathbf{1 4}}$ However, the above-mentioned analytical methods are costly and time consuming, require complicated pretreatment, consume large quantities of chemical agents and produce environmental pollution. Compared with the above methods, electrochemical techniques for detecting DA have attracted considerable attention because of the major advantage of the

Key Laboratory of Chemical Sensing \& Analysis in Universities of Shandong (University of Jinan), School of Chemistry and Chemical Engineering, University of Jinan, Jinan 250022, China. E-mail: chm_yfl518@163.com; Tel: +8653189736065 lower cost of the electrochemical instrument combined with fast detection, simplicity, facile operation and impressive costeffectiveness. With the development of novel functional materials, chemically modified electrodes are now widely used for the detection of dopamine in order to achieve lower detection limits and improve analytical sensitivity and selectivity. ${ }^{\mathbf{1 5 , 1 6}}$

Graphene oxide (GO) as a single-atom-thick and twodimensional carbon material has attracted remarkable interest in electrochemical analysis since it was first successfully manufactured in 2004. ${ }^{17-19}$ Graphene oxide is widely used in the sensing field for its excellent properties, such as huge surface area, unique structures and good chemical stability. GO is a layered flexible material with a wealth of functional groups, such as hydroxyl $(\mathrm{OH})$, epoxy (C-O-C), and carboxyl $(\mathrm{COOH})$ groups, on both the edges and basal planes. It can be easily functionalized to form GO-based materials. Guo et al. reported a high performance dopamine electrochemical sensor based on graphene oxide and Ag hybrid matrix electrodeposition L-lysine films, which was used to sensitively determine DA in the presence of ascorbic acid. ${ }^{20}$ Ghoreishi et al. fabricated a graphene oxide nano-sheet modified carbon paste electrode for the determination of dopamine in the presence of tyrosine. ${ }^{21}$ Thirumalraj et al. report the preparation of highly stable fullerene $\mathrm{C}_{60}$ decorated graphene oxide nanocomposite and its sensitive electrochemical detection of dopamine in rat brain and pharmaceutical samples. ${ }^{22}$ GObased materials modified electrodes are used for detection of 
DA because of three major reasons: biocompatibility, rapid electron transfer and low cost.

Ionic liquids (IL) are composed of large organic cations, such as pyridinium, phosphonium or imidazolium, and a relatively small anion, such $\mathrm{Cl}^{-}, \mathrm{Br}^{-}$, or larger complexes like tetrafluoroborate or hexafluorophosphate. Ionic liquids have some unique properties, such as high electric conductivity, low volatility and low toxicity. IL as a promising material have attracted great interest in the last few years. They have been widely used as electrode modifiers for constructed sensors, because of their viscosity, high conductivity and fairly wide electrochemical window. ${ }^{23-24}$ The excellent electrochemical conductivity of ILs could lead to more sensitive electrochemical responses, especially for the detection of trace components. Furthermore, ILs can be uniformly dispersed in graphene oxide owing to their good solvation properties.

In recent years, GO-based materials grafted onto noble metal nanoparticles have attracted considerable interest owing to their flexible structure, excellent catalytic capabilities and excellent electron transport properties. ${ }^{25}$ Recently, gold nanoparticles have been considered as an attractive candidate because of their huge specific surface area, excellent biocompatibility, good chemical stability and outstanding conductivity and catalytic properties. The unique two-dimensional crystal structure of GO acts as the substrate support for gold nanoparticles, which was attributed to the high electrocatalytic activities and extraordinary electronic transport properties. Meanwhile, composite materials prepared by gold nanoparticles dotted on graphene oxide as an enhanced sensing material have unparalleled electrochemical performance.

Herein, nanocomposites composed of gold nanoparticles anchored to ionic liquid functionalized graphene oxide were achieved by a simple one pot reaction. Ionic liquid as a linker attached gold nanoparticles and graphene oxide through electrostatic forces. Combining the advantageous features of gold nanoparticles and ionic liquid functionalized graphene oxide, the nanocomposites could incur synergy on electrochemical properties and thus improve the sensitivity of the nanomaterials. The prepared nanomaterials-modified glassy carbon electrode exhibited remarkable electrochemical catalytic activities towards dopamine. With a huge specific surface area and a large quantity of oxygen groups, GO shows good adsorption of dopamine. These oxidized areas on the GO plane break the long-range conjugated network and $\pi$-electron cloud, leading to a degradation of carrier mobility and conductivity. Ionic liquid functionalized graphene oxide could effectively prevent the agglomeration of graphene oxide, and the conductivity of the electrode could be enhanced and AuNPs have excellent load effects. The introduction of AuNPs brings not only electrochemical activity but also a certain biocompatibility. Meanwhile, AuNPs can effectively catalyze the electrochemical reaction of dopamine on the electrode surface. The electrochemical performance of the as-prepared sensor toward the oxidation of dopamine was explored in detail. The results indicated that the proposed sensor could achieve simple and efficient detection of dopamine.

\section{Experimental}

\subsection{Reagents and apparatus}

Dopamine, epinephrine, serotonin, ascorbic acid, uric acid, chloroauric acid and 1-butyl-3-methyl imidazole hydrobromide were purchased from Aladdin Industrial Corporation (Shanghai, China). Graphite powder, $\mathrm{H}_{2} \mathrm{SO}_{4}, \mathrm{HNO}_{3}, \mathrm{KCl}$, $\mathrm{KMnO}_{4}$ and ethanol were purchased from Sinopharm Group Chemical Reagent Co. without further purification. The $0.1 \mathrm{M}$ phosphate buffer solution (PBS) was prepared from $\mathrm{Na}_{2} \mathrm{HPO}_{4}$ and $\mathrm{NaH}_{2} \mathrm{PO}_{4}$. Chemicals used in the experiment were of analytical grade and all solutions were prepared with doubly distilled deionized water. Dopamine hydrochloride injection samples were purchased from the local hospital. The urine samples were collected from volunteers, and they were informed and agreed to follow the experiment.

All electrochemical measurements were performed on a $\mathrm{CHI}$ 660D electrochemistry workstation (Beijing Huake Putian Technology Co., Ltd. China). The classical three-electrode system was adopted. An $\mathrm{Ag} / \mathrm{AgCl}$ (saturated $\mathrm{KCl}$ ) electrode and a platinum electrode were used as the reference and counter electrodes, respectively. A bare or modified glassy carbon electrode (GCE) was used as the working electrode. Nanophase materials were characterized by scanning electron microscopy (S-2500, Hitachi High-tech International Trading Co., Ltd, Shanghai, China), X-ray diffraction (XRD, Focus D8 Bruker AXS Co. Ltd., Germany) and Fourier transform infrared spectroscopy (FTIR, Nicolet NEXUS-470, Thermo Nicolet, USA). Electrochemical impedance spectroscopy (EIS) was performed on a CHI 604D Electrochemical Workstation (Shanghai CH Instruments Inc., China). All the electrochemical experiments were carried out at room temperature.

\subsection{Synthesis of GO-IL-AuNPs}

The graphene oxide was synthesized from graphite power according to the modified Hummers' method. ${ }^{26,27}$ The GO was dispersed in ultrapure water to get a stock solution of $1.0 \mathrm{mg}$ $\mathrm{mL}^{-1} .10 \mathrm{mg}$ of 1-butyl-3-methyl imidazole hydrobromide was mixed with $10 \mathrm{~mL}$ of the GO dispersion. A GO-IL hybrid solution was obtained.

The gold nanoparticles were prepared using the method of sodium citrate reduction of chloroauric acid. ${ }^{28} 3.65 \mathrm{~mL}$ of chloroauric acid solution (0.01 M) was diluted into $150 \mathrm{~mL}$ and heated to micro-boiling. Then, $3.0 \mathrm{~mL}$ of sodium citrate was quickly added whilst heating and stirring. When the solution turned wine red, heating was stopped but stirring was continued until the solution was cooled to room temperature. The gold nanoparticles were stored in a refrigerator at $4{ }^{\circ} \mathrm{C} .10$ $\mathrm{mL}$ of gold nanoparticles was added into $20 \mathrm{~mL}$ of GO-IL hybrid solution, and ultrasonic treatment for $30 \mathrm{~min}$ produced the GOIL-AuNPs composite.

\subsection{Preparation of the modified electrodes}

Prior to the modification, the glassy carbon electrode (GCE) was polished with 0.3 and $0.05 \mu \mathrm{m}$ alumina slurries sequentially. Subsequently, the electrode was washed thoroughly with 


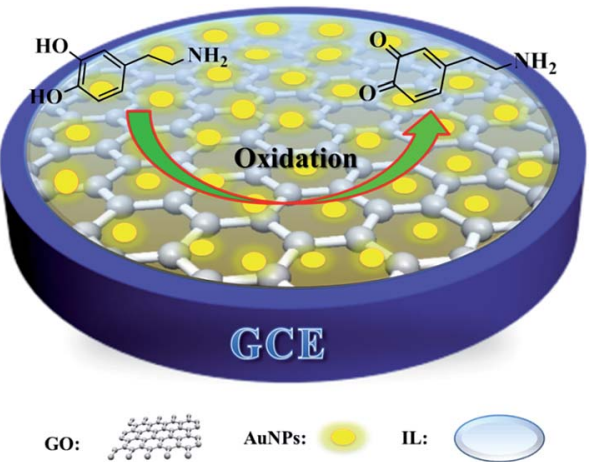

Scheme 1 Schematic illustration of the electrochemical sensors for the determination of DA.

ultrapure water and dried at room temperature. $5 \mu \mathrm{L}$ of the asprepared GO-IL-AuNPs composite suspension was modified to the surface of the polished GCE and the GO-IL-AuNPs nanofilms modified GCE (GO-IL-AuNPs/GCE) was thus obtained. The schematic illustration is shown in Scheme 1. As a control, GO modified GCE (GO/GCE) and GO-IL modified GCE (GO-IL/GCE) were also prepared.

\section{Results and discussion}

\subsection{Characterization of the nanocomposites}

The surface topography of (a) GO, (b) GO-IL, (c) AuNPs and (d) GO-IL-AuNPs nanocomposites was investigated by SEM measurement, and the SEM images are shown in Fig. 1. It is clear from Fig. 1a that the prepared graphene oxide displayed a typical crumpled and wrinkled lamellar structure. Fig. 1b presents an SEM image of GO-IL, which indicates that the surfaces of the GO sheets were uniformly coated with IL. Fig. 1c shows the SEM image of AuNPs, which show a particle diameter in the range of $12-18 \mathrm{~nm}$. The representative SEM image of GOIL-AuNPs is also shown in Fig. 1d, clearly illustrating that the uniform particle size gold nanoparticles are evenly decorated on the surface of the GO-IL, which suggests that GO-IL-AuNPs have been successfully synthesized.

The XRD patterns of (a) GO, (b) GO-IL, (c) GO-IL-AuNPs are given in Fig. 2A. It is obvious that graphene oxide displays a strong spectral peak at $10.3^{\circ}$ corresponding to the $\left(\begin{array}{lll}0 & 0 & 2\end{array}\right)$ diffraction crystalline plane. ${ }^{29}$ The XRD patterns of GO-IL are shown in Fig. 2Ab, and many diffraction peaks between $20^{\circ}$ and $30^{\circ}$ were attributed to the ionic liquid crystal structure ${ }^{30}$ and proven successful synthesis of GO-IL. The XRD pattern of the GO-IL-AuNPs composite shows the major diffraction peaks of $2 \theta$ at $38.4^{\circ}, 44.2^{\circ}$ and $65.3^{\circ}$, which corresponded to the $\left(\begin{array}{lll}1 & 1 & 1\end{array}\right),(2$ $00)$ and $\left(\begin{array}{lll}2 & 2 & 0\end{array}\right)$ planes of AuNPs and proved that AuNPs were successfully loaded on the surface of GO-IL.

To further investigate the formation of GO and GO-IL, FT-IR spectra were obtained. The FT-IR spectrum of GO is shown in Fig. 2Ba; the characteristic absorption bands at about 3400 , $1720,1640,1400$ and $1100 \mathrm{~cm}^{-1}$ correspond to the stretching vibrations of hydroxyl groups, carboxyl groups, alkene, carbon skeleton and epoxy, respectively. ${ }^{31}$ The spectrums of GO-IL

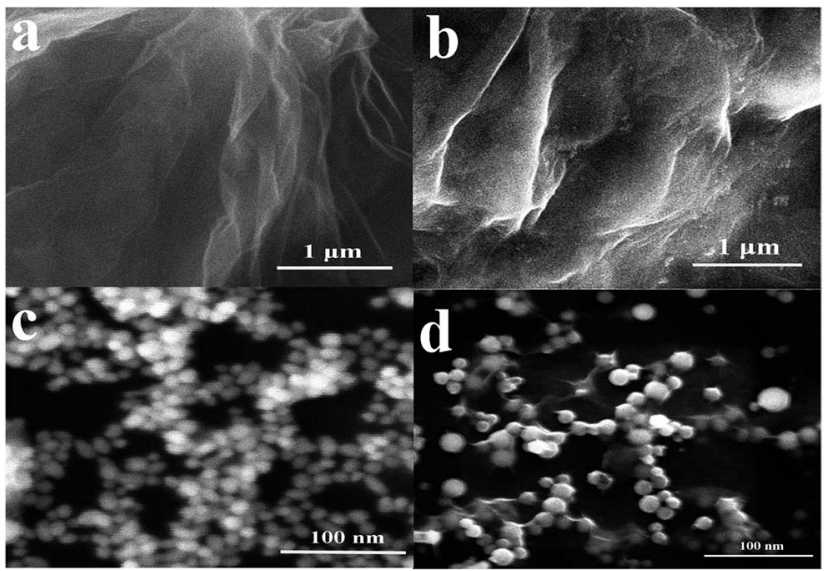

Fig. 1 The SEM images of (a) GO, (b) GO-IL, (c) AuNPs, (d) GO-ILAuNPs.

exhibit typical 1-butyl-3-methyl imidazole hydrobromide absorption features of $\mathrm{N}-\mathrm{H}$ stretching vibration $\left(3120 \mathrm{~cm}^{-1}\right)$, symmetric methylene stretching vibrations $\left(2870 \mathrm{~cm}^{-1}\right.$ and $\left.2960 \mathrm{~cm}^{-1}\right)$, C-N stretching vibration $\left(1630 \mathrm{~cm}^{-1}\right)$, imidazole ring skeletal vibration $\left(1570 \mathrm{~cm}^{-1}\right)$, imidazole ring deformation $\left(1060 \mathrm{~cm}^{-1}\right)$, and $\mathrm{C}-\mathrm{H}$ out-of plane bending vibration $(835$ $\mathrm{cm}^{-1}, 735 \mathrm{~cm}^{-1}$ and $\left.620 \mathrm{~cm}^{-1}\right) .{ }^{32,33}$ The results indicate that GO has been combined with the ionic liquid.

\subsection{Electrochemical studies}

Electrochemical impedance spectroscopy (EIS) is a powerful and effective method to monitor the interfacial properties of different modified electrodes. In the electrochemical impedance spectra, the semicircle part at high frequencies refers to the electron transfer controlled process and the linear part at low frequencies represents the diffusion controlled process. The semicircle diameter of the Nyquist plots is equal to the electron transfer resistance $\left(R_{\mathrm{et}}\right)$, which controls the electron transfer kinetics of the redox reaction at the electrode surface. $^{34,35}$ The electron transfer resistance can be directly calculated through the semicircle diameter. The electrochemical impedance of the bare GCE, GO/GCE, GO-IL/GCE and GO-IL-AuNPs/GCE was determined in $0.2 \mathrm{mM}\left[\mathrm{Fe}(\mathrm{CN})_{6}\right]^{3-/ 4-}$ solution containing $0.1 \mathrm{M} \mathrm{KCl}$ over the impedance frequency range from $1.0 \times 10^{5}$ to $0.1 \mathrm{~Hz}$. The Nyquist spectra are shown in Fig. 3A. It was easy to get the $R_{\mathrm{et}}$ of the bare GCE, GO/GCE, GO-IL/GCE and GO-IL-AuNPs/GCE, which were about $278 \Omega$, $1032 \Omega, 117 \Omega$ and $37 \Omega$, respectively. At the same time, the $R_{\text {et }}$ of these electrodes were in the following order: GCE $>$ GO/GCE $>$ GO-IL/GCE > GO-IL-AuNPs/GCE. This indicates that the resistance of electron transfer is effectively reduced owing to the introduction of IL and gold nanoparticles with excellent electron transfer properties. The GO-IL-AuNPs/GCE has good electro-catalytic performance for oxidation of DA.

The electrochemical behaviors of DA at different modified electrodes were investigated using cyclic voltammetry. Fig. 3B shows the CVs of the bare (a), GO (b), GO-IL (c) and GO-ILAuNPs (d) modified electrodes in 0.1 M PBS ( $\mathrm{pH}$ 7.0) 

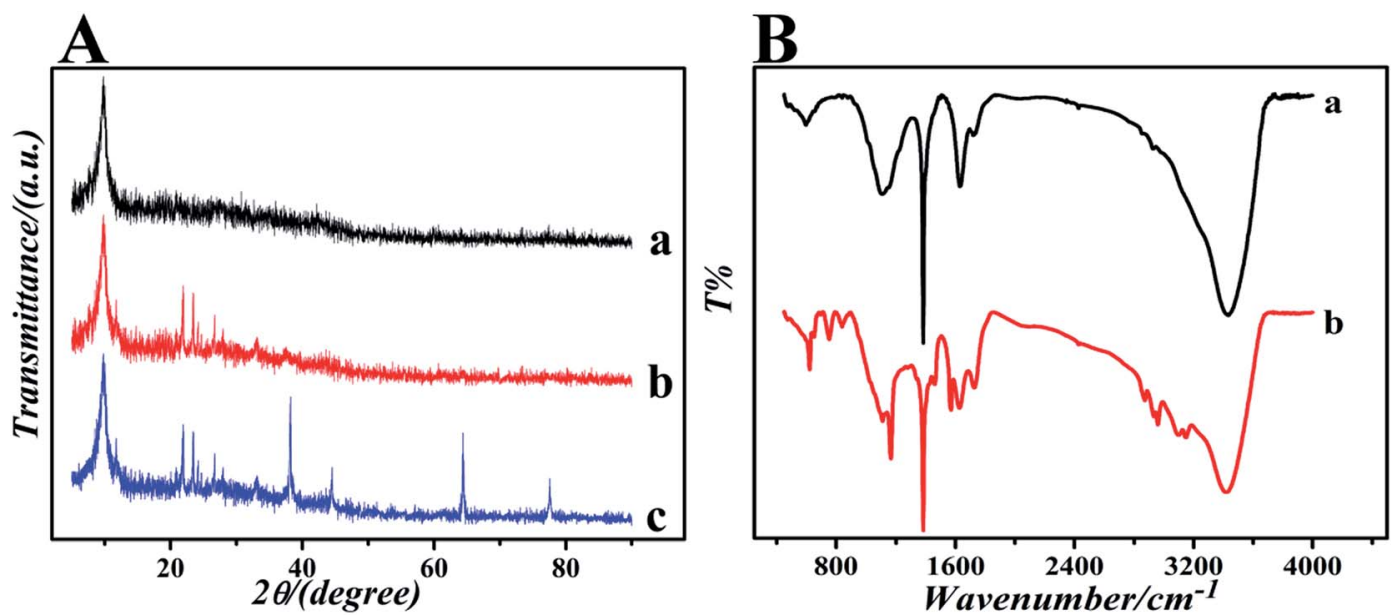

Fig. 2 (A) The XRD patterns of (a) GO, (b) GO-IL and (c) GO-IL-AuNPs. (B) The FT-IR spectra of (a) GO and (b) GO-IL.

containing $1 \mu \mathrm{M} \mathrm{DA}$ at a scan rate of $100 \mathrm{mV} \mathrm{s}^{-1}$. As can be seen from Fig. 3Ba, the bare GCE shows an obvious electrochemical oxidation peak for DA. Compared with the bare electrode, the DA electrochemical response signal intensity at the different modified electrodes was increased, which indicated that the modified materials have excellent electrical conductivity and electro-catalytic activity for DA oxidation. It is worth noting that a significant enhancement response signal was observed at the GO-IL-AuNPs/GCE (Fig. 3Bd), proving that the GO-IL-AuNPs nanocomposite has excellent electrical conductivity and electro-catalytic properties for oxidation of DA. Furthermore, the CVs responses of DA at the different electrodes were investigated in the presence of ascorbic acid. As shown in Fig. 3C, the CVs response of the bare GCE and GO modified GCE are only observed as one peak for the oxidation of AA and DA in PBS solution with $1 \mu \mathrm{M}$ DA and $40 \mu \mathrm{M} \mathrm{AA}$, indicating that the peak potential for AA and DA is indistinguishable. However, two welldefined oxidation peaks, corresponding to the oxidation of AA and DA, respectively, are clearly observed in the CV responses of the GO-IL and GO-IL-AuNPs modified GCEs, which provides the possibility of the detection of DA in the presence of ascorbic acid. The ionic liquid has a stronger electrical conductivity so may have better adsorption capacity for dopamine. Furthermore, the gold nanoparticles have better catalytic performance for dopamine.

\subsection{Optimization of the experimental conditions}

3.3.1. Influence of the amount of GO-IL-AuNPs nanocomposite suspension. The effect of the loading amount of GOIL-AuNPs on the electrode surface on the sensing properties was investigated. The relationship between the peak currents of DA and the amount of GO-IL-AuNPs on the GCE is shown in Fig. 4A. The response current value of $1 \mu \mathrm{M}$ DA gradually increased with the increase of the amount of GO-IL-AuNPs $\left(1 \mathrm{mg} \mathrm{mL}^{-1}\right)$ modified to the electrode surface from 3 to $6 \mu \mathrm{L}$. Nevertheless, a further increase in the volume of modification conversely caused a decrease in the response current of DA. Consequently, $6 \mu \mathrm{L}$ of GO-IL-AuNPs suspension was thought to be the optimal amount for the modified electrode.

3.3.2. Effect of solution $\mathbf{p H}$. The effect of $\mathrm{pH}$ on the electrochemical response of $1 \mu \mathrm{M}$ DA was investigated over the $\mathrm{pH}$ range from 4.5 to 7.5 and the results are shown in Fig. 4B. It is obvious that the peak current of DA increases continuously with
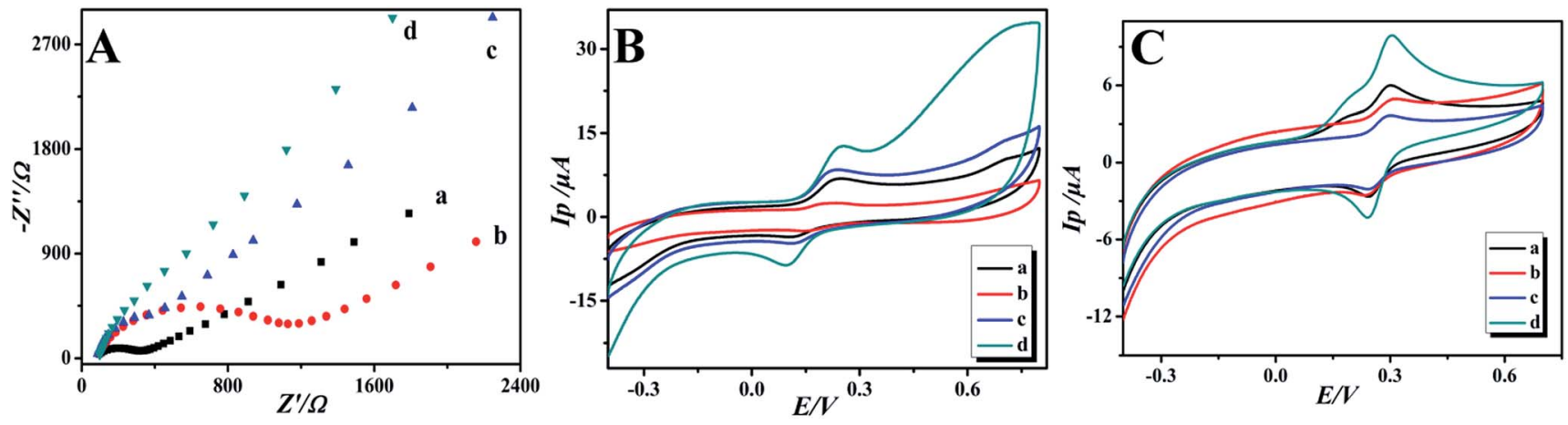

Fig. 3 Electrochemical impedance spectroscopy recorded in $0.2 \mathrm{mM} \mathrm{K}_{3}\left[\mathrm{Fe}(\mathrm{CN})_{6}\right]$ solution (A). Cyclic voltammograms showing the response of different modified electrodes to $1 \mu \mathrm{M} \mathrm{DA}$ (B); $1 \mu \mathrm{M}$ DA and $40 \mu \mathrm{M} \mathrm{AA}$ (C) in 0.1 M PBS. (a) GCE, (b) GO/GCE, (c) GO-IL/GCE, and (d) GO-IL-AuNPs/ GCE. 

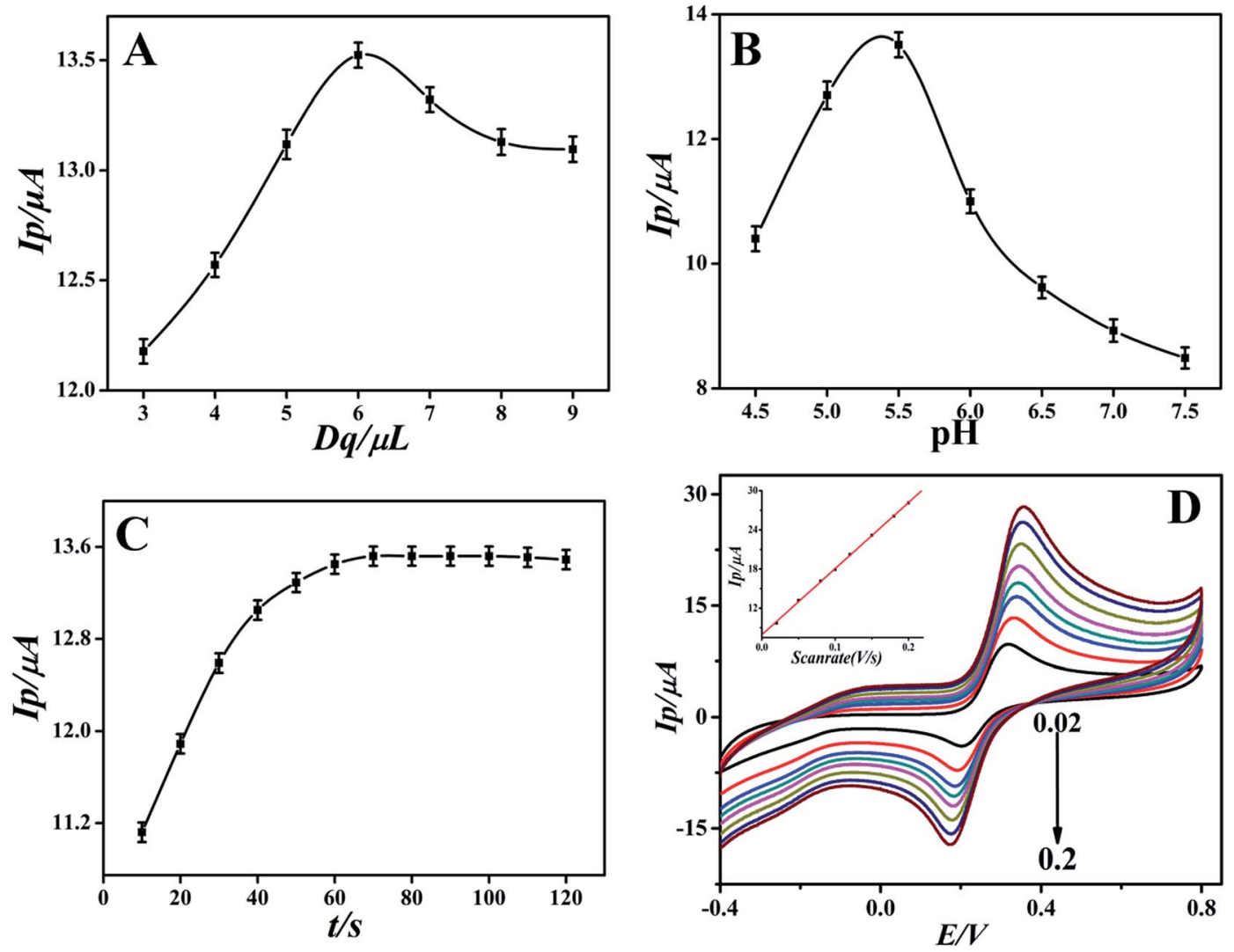

Fig. 4 (A) Effect of the volume of GO-IL-AuNPs. (B) Effect of pH value. (C) Effect of accumulation time. (D) CVs of GO-IL-AuNPs/GCE in 0.1 M PBS ( $\mathrm{pH}$ 5.5) with $10 \mu \mathrm{M}$ DA at different scan rates $\left(0.02,0.05,0.08,0.1,0.12,0.15,0.18\right.$, and $\left.0.2 \mathrm{~V} \mathrm{~s}^{-1}\right)$. Inset: the linear relationship between peak current and scan rate.

the increasing of the $\mathrm{pH}$ from 4.5 to 5.5 , and then gradually decreases on further increasing of the $\mathrm{pH}$. Therefore, $\mathrm{pH}$ of 5.5 was considered to be the optimal $\mathrm{pH}$ for the subsequent electrochemical experiments.

3.3.3. Effect of accumulation time. There is no doubt that the accumulation time will affect the sensitivity of the proposed sensor. The accumulation time can increase the amount of DA on the surface of the modified electrodes and thus improve the electrochemical response signal. Fig. 4C shows the relationship between the response current and the accumulation time. The response current gradually increased with the accumulation time increasing from 10 to $70 \mathrm{~s}$. When the accumulation time exceeded $70 \mathrm{~s}$, the peak current leveled off. Taking into account the sensitivity and efficiency of the sensor, $70 \mathrm{~s}$ was selected as the optimum accumulation time for the determination of DA.

3.3.4. Effect of scan rate. The effect of different scan rates on the electrochemical behavior of $10 \mu \mathrm{M}$ DA at the GO-ILAuNPs/GCE was investigated with cyclic voltammetry. The corresponding cyclic voltammograms are shown in Fig. 4D. It can be seen that the oxidation peak current increased gradually with the increase of scan rate. The linear relationship between the oxidation peak current and scan rate was obtained in the range of $0.02-0.2 \mathrm{~V} \mathrm{~s}^{-1}$ and the linear regression equation was $I_{\mathrm{p}}(\mu \mathrm{A})$ $=7.93005+101.3351 \nu\left(\mathrm{V} \cdot \mathrm{s}^{-1}\right), R=0.9994$. It indicated that the oxidation process of DA at the GO-IL-AuNPs/GCE surface was a typical adsorption controlled process rather than a diffusion controlled process.

\subsection{Analytical characteristics}

Under the optimal experimental conditions, the proposed sensor was employed for detecting various concentrations of DA

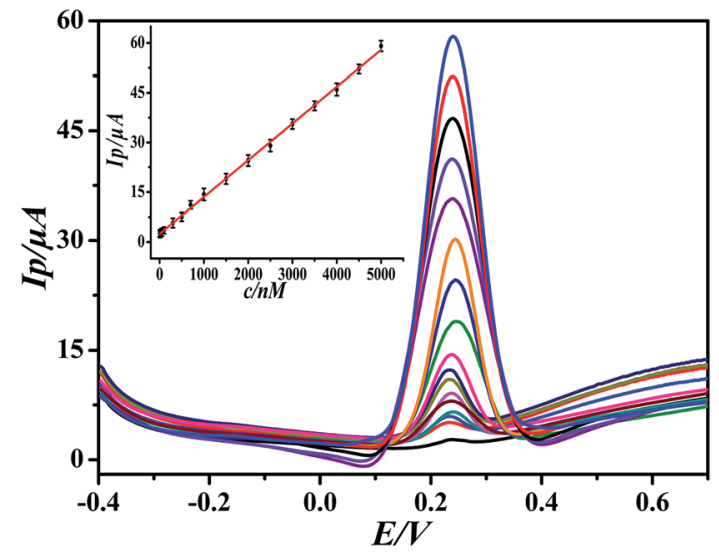

Fig. 5 Differential pulse voltammograms of different concentrations of DA at GO-IL-AuNPs/GCE ( $\mathrm{pH}=5.5)$. Inset: linear calibration curve in the range $7 \mathrm{nM}$ to $5 \mu \mathrm{M} \mathrm{DA}$. 
Table 1 Comparison of the performances of some sensors for the detection of DA

\begin{tabular}{lllll}
\hline Modified electrodes & Method & $\begin{array}{l}\text { Linear } \\
\text { range (nM) }\end{array}$ & $\begin{array}{l}\text { Detection } \\
\text { limit (nM) }\end{array}$ & References \\
\hline Pt/graphene/GCE & DPV & $30-8130$ & 30 & 36 \\
SWCNTs/GCE & DPV & $10-200$ & 15 & 37 \\
NCQDs/GCE & DPV & $10-1000$ & 10 & 38 \\
GNR/GCE & SWV & $10-100$ & 5.5 & 39 \\
PtNPs-MWCNT/GCE & DPV & $60-2030$ & 50 & 40 \\
AuNPs@MIES/GE & DPV & $20-540$ & 7.8 & 41 \\
GO-IL-AuNPs/GCE & DPV & $7-5000$ & 2.3 & This work
\end{tabular}

in $0.1 \mathrm{M}$ PBS by more sensitive differential pulse voltammetry. The electrochemical response of DA may follow the two proton transfer process through the ECE mechanism on the modified electrode. DA oxidation at the surface of the modified electrode was enhanced by diffusion and adsorption through graphene oxide and the ionic liquid, to make contact with the electrode surface easy and facilitate the electron transfer. The electrocatalytic performance of the GO-IL-AuNPs/GCE for DA is shown in Fig. 5. The inset of Fig. 5 displays the linear relationship between response current and the DA concentration in the range of $7 \mathrm{nM}$ to $5 \mu \mathrm{M}$. The linearity regression equation was $I_{\mathrm{p}}$ $(\mu \mathrm{A})=0.0111 c(\mathrm{nM})+2.4126(R=0.9994)$. The detection limit of DA was calculated to be $2.3 \mathrm{nM}(\mathrm{S} / \mathrm{N}=3)$. The above results indicate that the reported GO-IL-AuNPs/GCE has excellent electro-catalytic ability for DA oxidation. As a comparison, the analysis performance of other dopamine sensors is listed in Table 1. It can be seen that the proposed sensor demonstrated better performance compared to other sensors.

To ensure the repeatability of the proposed sensor, repeated experiments were operated in PBS containing $1 \mu \mathrm{M}$ DA with the same modified electrode. The results of ten measurements showed a relative standard deviation (RSD) of $2.8 \%$. Additionally, the reproducibility of the sensor was estimated by using six sensors prepared in the same way for determination of the same DA solution. The relative standard deviation (RSD) was 3.1\%. The storage stability of the proposed sensor was also evaluated. When the sensor was stored in the environment for two weeks, the response current was not significantly weakened and remained at $96.5 \%$ of its initial current response, and $92.2 \%$ of the initial current response remained after four weeks. These results indicated that the proposed sensor had good repeatability, reproducibility and stability.

In order to evaluate the potential of interference during DA detection, possible interfering compounds such as AA, glucose, uric acid (UA), epinephrine (EP) and serotonin (5-HT) were used. It was found that 40 -fold concentrations of AA, uric acid, and epinephrine and 100-fold concentrations of glucose and serotonin (5-HT) had no obvious influence on the response current of $1 \mu \mathrm{M}$ DA with deviations below $\pm 5 \%$. Besides, some 200-fold concentration of excess ions such as $\mathrm{Mg}^{2+}, \mathrm{Al}^{3+}, \mathrm{Cl}^{-}$, $\mathrm{SO}_{4}{ }^{2-}$ and $\mathrm{NO}_{3}{ }^{-}$had no influence on the DA response signal. These results demonstrate that GO-IL-AuNPs/GCE has good selectivity towards DA.
Table 2 Determination of DA in actual samples $(n=6)$

\begin{tabular}{lllll}
\hline Sample & $\begin{array}{l}\text { Added } \\
(\mu \mathrm{M})\end{array}$ & $\begin{array}{l}\text { Found } \\
(\mu \mathrm{M})\end{array}$ & $\begin{array}{l}\text { RSD } \\
(\%)\end{array}$ & $\begin{array}{l}\text { Recovery } \\
(\%)\end{array}$ \\
\hline \multirow{2}{*}{ Urine } & 0 & - & - & - \\
& 0.5 & 0.51 & 3.7 & 102 \\
& 1.0 & 0.98 & 3.2 & 98 \\
\multirow{4}{*}{ Injection } & 1.5 & 1.52 & 3.6 & 101 \\
& 0 & 1.02 & 2.1 & - \\
& 1.0 & 2.02 & 2.3 & 100 \\
& 1.5 & 2.57 & 2.2 & 102 \\
& 2.0 & 3.00 & 2.5 & 99
\end{tabular}

\subsection{Detection of dopamine in real samples}

In order to verify the reliability of the proposed sensor, the GOIL-AuNPs/GCE was used for the analysis of DA in urine and dopamine hydrochloride injection samples by differential pulse cyclic voltammetry. The standard addition method was employed to analyze the content of DA in these actual samples. The results of the analysis are summarized in Table 2 . As can be seen from the results in Table 2, the proposed sensor has good accuracy of detection with recoveries in the range from $98 \%$ to $102 \%$ and a relative standard deviation below $4 \%$. The above sample analysis results indicate that the proposed sensor is very feasible and sensitive enough to detect trace dopamine in real samples. The sample was characterized by high performance liquid chromatography after 50 cycles. As a result, dopamine was not detected, which indicates that the DA had reacted completely after 50 cycling tests.

\section{Conclusions}

In this work, a novel platform of an electrochemical sensor based on 1-butyl-3-methylimidazolium cationic groups functionalized graphene oxide film loaded gold nanoparticles was constructed. The ionic liquid has good biocompatibility and strong electron transfer performance. The loading of gold nanoparticles significantly enhanced the catalytic performance of the complexes. The electrochemical properties were evaluated toward the electro-oxidation of dopamine. The interference experiments showed that some common interferents, such as uric acid, epinephrine, and ascorbic acid, caused no interference in the determination of dopamine. Moreover, the proposed sensor has good stability, excellent selectivity and good electrocatalytic activity. Under the optimal conditions, a stable linear relationship was observed between the response current and the concentration of DA in the range from $7 \mathrm{nM}$ to 5 $\mu \mathrm{M}$ with a limit of detection $2.3 \mathrm{nM}$. The as-prepared sensor was employed to analyze real samples and achieved satisfactory results.

\section{Acknowledgements}

This work was supported by the Shandong Provincial Natural Science Foundation of China (No. ZR2012BM020) and the 
Scientific and technological development Plan Item of Jinan City in China (No. 201202088).

\section{References}

1 C. Deng, J. Chen, M. Wang, et al., A novel and simple strategy for selective and sensitive determination of dopamine based on the boron-doped carbon nanotubes modified electrode, Biosens. Bioelectron., 2009, 24, 2091-2094.

2 B. E. K. S. M. Pandurangachar, U. Chandra, O. Gilbert and B. S. Sherigara, Simultaneous determination of dopamine, ascorbic acid and uric acid at poly (Patton and Reeder's) modified carbon paste electrode, Int. J. Electrochem. Sci., 2009, 4, 672-683.

3 R. Zhang, G. D. Jin, D. Chen and X. Y. Hu, Simultaneous electrochemical determination of dopamine, ascorbic acid and uric acid using poly(acid chrome blue K) modified glassy carbon electrode, Sens. Actuators, B, 2009, 138, 174-181.

4 W. M. Caudle, R. E. Colebrooke, P. C. Ernson and G. W. Miller, Altered vesicular dopamine storage in Parkinson's disease: a premature demise, Trends Neurosci., 2008, 31, 303-308.

5 P. Y. Chen, R. Vittal, P.-C. Nien and K.-C. Ho, Enhancing dopamine detection using a glassy carbon electrode modified with MWCNTs, quercetin, and Nafion, Biosens. Bioelectron., 2009, 24, 3504-3509.

6 Z. Guo, M. L. Seol, M. S. Kim, J. H. Ahn, Y. K. Choi, J. H. Liu and X. J. Huang, Sensitive and selective electrochemical detection of dopamine using an electrode modified with carboxylated carbonaceous spheres, Analyst, 2013, 138, 2683-2690.

7 Z. Herrasti, F. Martínez and E. Baldrich, Electrochemical detection of dopamine using streptavidin-coated magnetic particles and carbon nanotube wiring, Sens. Actuators, B, 2014, 203, 891-898.

8 J. Y. Park, S. W. Myung, I. S. Kim, D. K. Choi, S. J. Kwon and S. H. Yoon, Simultaneous Measurement of Serotonin, Dopamine and Their Metabolites in Mouse Brain Extracts by High-Performance Liquid Chromatography with Mass Spectrometry Following Derivatization with Ethyl Chloroformate, Biol. Pharm. Bull., 2013, 36, 252-258.

9 C. G. Qian, S. Zhu, P. J. Feng, Y. L. Chen, J. C. Yu, X. Tang, Y. Liu and Q. D. Shen, Conjugated Polymer Nanoparticles for Fluorescence Imaging and Sensing of Neurotransmitter Dopamine in Living Cells and the Brains of Zebrafish Larvae, ACS Appl. Mater. Interfaces, 2015, 7, 18581-18589.

10 Q. Zhu, Y. L. Chen, W. F. Wang, H. G. Zhang, C. L. Ren, H. L. Chen and X. G. Chen, A sensitive biosensor for dopamine determination based on the unique catalytic chemiluminescence of metal-organic framework HKUST-1, Sens. Actuators, B, 2015, 210, 500-507.

11 V. Carrera, E. Sabater, E. Vilanova and M. A. Sogorb, A simple and rapid HPLC-MS method for the simultaneous determination of epinephrine, norepinephrine, dopamine and 5-hydroxytryptamine: application to the secretion of bovine chromaffin cell cultures, J. Chromatogr. B: Anal. Technol. Biomed. Life Sci., 2007, 847, 88-94.
12 S. R. Wallenborg, L. Nyholm and C. E. Lunte, End-Column Amperometric Detection in Capillary Electrophoresis: Influence of Separation-Related Parameters on the Observed Half-Wave Potential for Dopamine and Catechol, Anal. Chem., 1999, 71, 544-549.

13 E. Causse, A. Pradelles, B. Dirat, A. Negre-Salvayre, R. Salvayre and F. Couderc, Simultaneous determination of allantoin, hypoxanthine, xanthine, and uric acid in serum/ plasma by CE, Electrophoresis, 2007, 28, 381-387.

14 S. Kumbhat, D. R. Shankaran, S. J. Kim, K. V. Gobi, V. Joshi and N. Miura, Surface plasmon resonance biosensor for dopamine using D3 dopamine receptor as a biorecognition molecule, Biosens. Bioelectron., 2007, 23, 421-427.

15 Z. J. Yang, X. C. Huang, J. Li, Y. C. Zhang, S. H. Yu, Q. Xu and X. Y. Hu, Carbon nanotubes-functionalized urchin-like $\operatorname{In}_{2} \mathrm{~S}_{3}$ nanostructure for sensitive and selective electrochemical sensing of dopamine, Microchim. Acta, 2012, 177, 381-387.

16 J. Li, J. Yang, Z. J. Yang, Y. F. Li, S. H. Yu, Q. Xu and X. Y. Hu, Graphene-Au nanoparticles nanocomposite film for selective electrochemical determination of dopamine, Anal. Methods, 2012, 4, 1725-1728.

17 K. S. Novoselov, A. K. Geim, S. V. Morozov, D. Jiang, Y. Zhang, S. V. Dubonos, I. V. Grigorieva and A. A. Firsov, Electricfield effect in atomically thin carbonfilms, Science, 2004, 306, 666-669.

18 S. B. Yang, X. L. Feng, L. J. Zhi, Q. A. Cao, J. Maier and K. Mullen, Nanographene-constructed hollow carbon spheres and their favorable electroactivity with respect to lithium storage, Adv. Mater., 2010, 22, 838-842.

19 E. J. H. Lee, L. J. E. Zhi, M. Burghard, K. Mullen and K. Kern, Electrical properties and photoconductivity of stackedgraphene carbon nanotubes, Adv. Mater., 2010, 22, 18541857.

20 Z. Guo, G. Q. Huang, J. Li, Z. Y. Wang and X. F. Xu, Graphene oxide-Ag/poly-L-lysine modified glassy carbon electrode as an electrochemical sensor for the determination of dopamine in the presence of ascorbic acid, J. Electroanal. Chem., 2015, 759, 113-121.

21 S. M. Ghoreishi, M. Behpour, M. Mortazavi and A. Khoobi, Fabrication of a graphene oxide nano-sheet modified electrode for determination of dopamine in the presence of tyrosine: a multivariate optimization strategy, J. Mol. Liq., 2016, 215, 31-38.

22 B. Thirumalraj, S. Palanisamy, S. M. Chen and B. S. Lou, Preparation of highly stable fullerene $\mathrm{C}_{60}$ decorated graphene oxide nanocomposite and its sensitive electrochemical detection of dopamine in rat brain and pharmaceutical samples, J. Colloid Interface Sci., 2016, 462, 375-381.

23 H. Karimi-Maleh, F. Tahernejad-Javazmi, N. Atar, M. L. Yola, V. K. Gupta and A. A. Ensafi, A novel DNA biosensor based on a pencil graphite electrode modified with polypyrrole/ functionalized multiwalled carbon nanotubes for determination of 6-mercaptopurine anticancer drug, Ind. Eng. Chem. Res., 2015, 54, 3634-3639.

24 A. Baghizadeh, H. Karimi-Maleh, Z. Khoshnama, A. Hassankhani and M. Abbasghorbani, Voltammetric 
sensor for simultaneous determination of vitamin $\mathrm{C}$ and vitamin $\mathrm{B} 6$ in food samples using $\mathrm{ZrO}_{2}$ nanoparticle/ionic liquids carbon paste electrode, Food Anal. Method, 2015, 8, 549-557.

25 X. M. Wang, M. X. Lu, H. Wang, Y. F. Pei, H. H. Rao and X. Z. Du, Three-dimensional graphene aerogelsmesoporous silica frameworks for superior adsorption capability of phenols, Sep. Purif. Technol., 2015, 153, 7-13.

26 W. S. Hummers and R. E. Offeman, Preparation of graphitic oxide, J. Am. Chem. Soc., 1958, 80, 1339.

27 X. J. Li, X. J. Wang, L. L. Li, H. M. Duan and C. N. Luo, Electrochemical sensor based on magnetic graphene oxide@gold nanoparticles-molecular imprinted polymers for determination of dibutyl phthalate, Talanta, 2015, 131, 354-360.

28 N. I. Kovtyukhova, P. J. Ollivier, B. R. Martin, T. E. Mallouk, S. A. Chizhik, E. V. Buzaneva and A. D. Gorchinskiy, Layer-byLayer Assembly of Ultrathin Composite Films from MicronSized Graphite Oxide Sheets and Polycations, Chem. Mater., 1999, 11, 771-778.

29 Y. Li, W. Gao, L. Ci, C. Wang and P. M. Ajayan, Catalytic performance of $\mathrm{Pt}$ nanoparticles on reduced graphene oxide for methanol electro-oxidation, Carbon, 2010, 48, 1124-1130.

30 C. W. Liew, K. H. Arifin, J. Kawamura, Y. Iwaib, S. Ramesh and A. K. Arof, Electrical and structural studies of ionic liquid-based poly(vinyl alcohol) proton conductors, J. NonCryst. Solids, 2015, 425, 163-172.

31 C. Nethravathi, T. Nisha, N. Ravishankar, C. Shivakumara and M. Rajamathi, Graphene-nanocrystalline metal sulphide composites produced by a one-pot reaction starting from graphite oxide, Carbon, 2009, 47, 2054-2059.

32 X. M. Chen, T. Q. Ren, M. Ma, Z. G. Wang and G. Q. Zhan, Voltammetric sensing of bisphenol A based on a singlewalled carbon nanotubes/poly\{3-butyl-1-[3-(N-pyrrolyl) propyl] imidazolium ionic liquid $\}$ composite film modified electrode, Electrochim. Acta, 2013, 111, 49-56.

33 A. M. Gurban, L. Rotariu, M. Baibarac, I. Balto and C. Bala, Sensitive detection of endocrine disrupters using ionic liquid-single walled carbon nanotubes modified screenprinted based biosensors, Talanta, 2011, 85, 2007-2013.

34 Z. Wang, K. Shang, J. Dong, Z. Cheng and S. Ai, Electrochemical immunoassay for subgroup $\mathrm{J}$ of avian leukosis viruses using a glassy carbon electrode modified with a film of poly (3-thiophene boronic acid), gold nanoparticles, graphene and immobilized antibody, Microchim. Acta, 2012, 179, 227-234.

35 R. Ehret, W. Baumann, M. Brischwein, A. Schwinde, K. Stegbauer and B. Wolf, Monitoring of cellular behaviour by impedance measurements on interdigitated electrode structures, Biosens. Bioelectron., 1997, 12, 29-41.

36 C. L. Sun, H. H. Lee, J. M. Yang and C. C. Wu, The simultaneous electrochemical detection of ascorbic acid, dopamine, and uric acid using graphene/size-selected Pt nanocomposites, Biosens. Bioelectron., 2011, 26, 3450-3455.

37 S. Alwarappan, G. Liu and C. Z. Li, Simultaneous detection of dopamine, ascorbic acid, and uric acid at electrochemically pretreated carbon nanotube biosensors, Nanomedicine, 2010, 6, 52-57.

38 G. Jiang, T. T. Jiang, H. J. Zhou, J. M. Yao and X. D. Kong, Preparation of N-doped carbon quantum dots for highly sensitive detection of dopamine by an electrochemical method, RSC Adv., 2015, 5, 9064-9068.

39 Z. Jia, J. Liu and Y. Shen, Fabrication of a templatesynthesized gold nanorod-modified electrode for the detection of dopamine in the presence of ascorbic acid, Electrochem. Commun., 2007, 9, 2739-2743.

40 Z. Dursun and B. Gelmez, Simultaneous determination of ascorbic acid, dopamine and uric acid at Pt nanoparticles decorated multiwall carbon nanotubes modified GCE, Electroanalysis, 2010, 22, 1106-1114.

41 C. Xue, Q. Han, Y. Wang, J. H. Wu, T. T. Wen, R. Y. Wang, J. L. Hong, X. M. Zhou and H. J. Jiang, Amperometric detection of dopamine in human serum by electrochemical sensor based on gold nanoparticles doped molecularly imprinted polymers, Biosens. Bioelectron., 2013, 49, 199-203. 